\title{
Investigation on Energy Distribution in Steady and Unsteady Flow Instabilities through a Bend Square Pipe
}

\author{
Mohammad Sanjeed Hasan ${ }^{1}$, Sabrina Rashid ${ }^{2}$, Shamsun Naher Dolon ${ }^{3}$, Ratan Kumar Chanda ${ }^{3}$, \\ Muhammad Minarul Islam ${ }^{1}$, Rabindra Nath Mondal $^{3}$, Giulio Lorenzini ${ }^{4}$ \\ ${ }^{1}$ Department of Mathematics, Bangabandhu Sheikh Mujibur Rahman Science and Technology University, Gopalganj- \\ 8100, Bangladesh, e-mail: sanjeedlhasan@gmail.com; minarul_math@yahoo.com \\ ${ }^{2}$ Department of Applied Mathematics, University of Dhaka, Dhaka-1100, Bangladesh, e-mail: \\ sabrinarashid374@gmail.com \\ ${ }^{3}$ Department of Mathematics, Jagannath University, Dhaka-1100, Bangladesh, e-mail: dolonjnu02@ gmail.com; \\ ratan222003@gmail.com; rnmondal@jnu.ac.bd
}

${ }^{4}$ Department of Engineering and Architecture, University of Parma, Parma 43124, Italy, e-mail: giulio.lorenzini@unipr.it

\begin{tabular}{l} 
Article Info \\
\hline Article history: \\
Received April 15, 2021 \\
Revised May 13, 2021 \\
Accepted May 25, 2021 \\
\hline
\end{tabular}

\section{Keywords:}

Curved pipe,

Steady solution,

Unsteady solution,

Energy Distribution,

Experimental validation.

\begin{abstract}
Fluid flow analysis through a bend pipe is extensively conducted in practical and cell separation operations. It is observed that flow behaviors in the bend pipe are influenced by some parameters such as curvature, aspect ratio, etc. As a result, various phenomena, steady solution branches, unsteady solutions, energy transfer are changed. In this paper, the acts of flows are performed together for fixed curvature, $\delta=0.2$, and Prandtl number, $\operatorname{Pr}=7.0$ (water). Here, for a wide variety of Dean numbers $(100 \leq D n \leq 1000)$ and three fixed Grashof numbers, $G r=100,500$, and 1000; time-independent solutions with linear stabilities are investigated first where only the first steady branch exhibits linear stability out of two steady solution branches obtained. Then, different flow transitions between the required range of Dean numbers $(D n)$ and several Grashof numbers $(G r)$ are investigated using time-dependent solutions. Power spectrum density (PSD) is further revealed in order to gain a deeper understanding of periodic and multi-periodic flows. Flow velocity contours including axial flow (AF) and secondary flow (SF) and their temperature profiles (TP) are also exposed. The SFs reveal that two- to four vortex flows are produced due to the turning of steady branch and the flow instabilities. Furthermore, the energy transfer between the cooled and heated sidewalls of the pipe is calculated. Finally, a link between centrifugal and body force with the energy transfer has been shown in this research which reveals that the fluid has merged that certainly rises the overall energy transfer.
\end{abstract}

Copyright (C) 2020 Regional Association for Security and crisis management and European centre for operational research. All rights reserved.

\section{Corresponding Author:}

Mohammad Sanjeed Hasan,

Department of Mathematics, Bangabandhu Sheikh Mujibur Rahman Science and Technology University, Gopalganj-8100, Bangladesh

Email: sanjeedlhasan@gmail.com

\section{Introduction}

Working on bend pipe flows has not only started from today but has continued from a century ago. It is extensively utilized in mechanical and biomedical engineering department such as fluid transportation, fluid amplifiers, heat exchangers, rocket engine, coil steam generators, ventilators, nuclear reactors, thermo siphons, human vein and arteries, tumor cells, etc. The governing equations for the bend pipe were invented by Dean (1927) first. He expressed that the flows through the duct had been influenced by curvature which was known 
as centrifugal force later. On account of this, two vortex flow patterns are found and at a critical point, they are converted into four vortex flow. These types of the vortex are known as Dean vortex. Presently, various types of curved duct/pipe are used by many scholars. Some distinguished papers on curved duct are referenced by Mondal et al. (2006) and Yanase et al. (2005) (square and rectangular duct), Fiola and Agarwal (2015) (S shape duct), Anand and Sandeep (2010) (Y shape duct), Chandratilleke et al. (2013) (elliptical duct), Ferdows et al. (2007) (helical duct).

There are various types of flow observations are investigated in the bend pipe. One of them is the bifurcation structure. Recently, Watanabe and Yanase (2013) obtained various bifurcation structures for the threedimensional case and found a connection between the branches. Bifurcation diagram from small to large aspect ratio for different aspect ratios have been also calculated by Mechane (2010). Using Euler-Newton continuation method, Yang and Wang (2001) pointed out the position of the symmetric and asymmetric solution of branches. They tried to find out the dynamic responses of the branch. A complete study on bifurcation has been performed by Mondal et al. (2007). Two types of bifurcation such as Holf and Pitchfork bifurcation and the curvature effect to form a bifurcation have been expressed by them. They further examined the transitional phenomena through the curved square duct for the isothermal case. For both rotating and non-rotating duct, Hasan et al. (2019a, 2019b) used spectral method and found out two and four steady branches in the Taylor and Dean plane respectively. Analysis of bifurcation in partially wetting liquids for different values of the equilibrium contact angle has been conducted by Lin et al. (2016). The change of bifurcation structures with respect to the electric parameters has been demonstrated by Lin et al. (2017) for two-dimensional inviscid and incompressible fluid. The change of bifurcation in multi-vortex secondary flow structures have been visualized experimentally by Leong et al. (2009) where they provided particle image velocimetry method. Gelfgat (2020) exposed the Dean and Reynold number instabilities in the steady solution for helical pipes.

Time evolution calculations for different aspect ratios have been investigated by Mondal et al. (2013). To justify the oscillating flows they also visualized phase space. Czajkowski et al. (2020) experimentally narrated the influence of flow transition for various rotational speeds, heat flux, filling ratios through a pipe. Canton et al. (2016) reported unsteady flow characteristics in a turbulent channel for small Reynold nubers. Dolon et al. (2019) tried to detect the oscillating behavior through the curved duct with increasing the pressure gradient parameter. Two to ten vortex secondary flows have been acquired in their study for the oscillating flows. Dynamical responses of the unsteady behavior in the steady symmetric and asymmetric solution branches have been reported separately by Liu and Wang (2019). Arpino et al. (2015) analyzed the effects of thermal transient behaviors for Rayleigh number, Darcy number and aspect ratio in the porous and partially porous cavities. Zhang et al. (2019) guided their investigation on the flow around four equispaced cylinders to find out the unsteady flow characteristics with respect to two parameters including lift and drag coefficient. Unsteady behaviors for large aspect ratio at different points of the bifurcations have been presented by Yanase et al. (2002). The time-dependent behavior of the rectangular duct of aspect ratio 0.5 has been elaborated by Mondal et al. (2015) for different Grashof numbers and large Dean numbers. Bifurcation structures as well as the unsteady flow characteristics of the circular duct have been observed by Islam et al. (2019).

Fluid characteristics through the ducts investigations play a very important role in many biological and engineering applications. Two-dimensional flow regimes between two square cylinders for different Reynolds numbers and gap ratios have been disclosed by Nazeer et al. (2019). By validating the fluid flow found by computational fluid dynamics with the particle image velocimetry measurements, Li et al. $(2016,2017)$ depicted the Dean instabilities in the mass and energy transfer for rectangular bend channel. Gao et al. (2020) depicted the isotherms and the hydraulic flow velocity behavior for the horizontal duct. Fomicheva et al. (2019) conducted Thomas algorithm to present the flow velocities through a funnel. Two-dimensional axial and secondary flow characteristics for non-rotating and rotating duct has been illustrated by Hasan et al. (2020a, $2020 \mathrm{~b}$, and 2020c). They showed that the axial velocities were more affected at the rotating curved duct than the non-rotating curved duct. Wang et al. (2018) explained the variation of Dean vortices for different rotations and gap ratios in the rotating cylinder. Kim et al. (2011) showed that the fluid flow has not significantly influenced by the variation of Reynold number with the development of friction factor through the helical pipes. Bu et al. (2015) elucidated the variational characteristics of streamlines in the secondary flow through the curved annular ducts. Chen et al. (2020) examined the temperature distribution at different inlets in the presence of Joule-Thomson parameters through the helically coiled tubes. Arvanitis et al (2018) numerically carried out the influence between the streamlines and isotherms for several Dean and Reynold numbers. Soltanipour et al (2020) examined the effect of the magnetic field and entropy generation in the streamlines at different angles of the curved pipe. Sultana et al. (2019) presented the impacts of flow velocities and heat transfer in bifurcation and unsteady flow structures.

Heat transfer calculation from the curved square duct is another important phenomenon for the researchers. Chandratilleke et al. (2013) offered a numerical and experimental investigation of flow-through both rectangular and elliptical channels with heat transfer effects. Hashemi et al. (2018) discussed the fluctuation of

Investigation on energy distribution in steady and unsteady flow instabilities through a bend square pipe (Mohammad Sanjeed Hasan) 
the axial flow with the change of time through a finite length curved pipe. Rahmani et al. (2019) represented the isotherms contours where they employed finite Fourier transform and the method to calculate the heat transfer. Bhuyan and Giri (2021) analyzed the energy distribution and entropy generation of the air-water flow through the vertical tube where they have further shown that the second law has a significant effect on heat transfer. Thermal buoyancy effect and mean Nusselt number in the curved duct have been calculated by Mokeddem et al. (2019) for different Dean numbers and aspect ratios. Praks and Chanda et al. (2020) reported the change of energy distribution as well as flow velocity effects for increasing $\operatorname{Tr}$ for both positive and negative direction, where Dolon et al. (2021) conducted a nearly identical study for a non-circular rectangular channel. Hasan et al. (2021) illustrated the changing of overall heat flux energy for increasing $T r$. Ghobadi and Muzychka (2015) interpreted a relation between heat transfer and pressure drops in Newtonian fluid for curved and coiled circular tubes. Manesh et al. (2019) analytically solved the heat transfer in composite canonical shells and shown it from different angles in the Heat flux vs. Mean temperature plane. The rate of heat transfer through the curved annular pipe with showing a connection between the friction factor, Nusselt number and Dean number, has been elucidated by Nobari et al. (2009). Arpino et al. (2014) exhibited the porous convection and the porous cavities of the flow for different aspect ratios, Rayleigh and Darcy number. Razavi et al. (2015) have driven the finite volume method to depict the convection in the curved rotating duct where they have applied the second laws of thermodynamics. Effects heat transfer in the axial flow in the inner and outer walls through rectangular pipe have been explained by Norouzi et al. (2011). Heat transfer effects in the Dean number vs. Nusselt number plane has been also detected by Mondal et al. (2017) for large aspect ratio. They also established a relation between the centrifugal force and heating induced buoyancy force together with drawing a vortex diagram. Hasan et al. (2019c, 2019d) adopted the Centrifugal and Coriolis parameter to discuss the cause of increasing the heat transport as well as temperature gradients in the revolving and non-revolving coiled tubes. They moreover pointed out the heat transfer effects in the flow transitions and the steady flows.

Among the studies, scholars have published their paper on the bifurcation, unsteady flow characteristics and heat transfer separately. But it is a little hard to understand all things when a paper is related to the other ones. Therefore, the article aims to analyze and explain the change of flow vorticity and the energy distribution in time-dependent and independent solutions.

\section{Mathematical Model of the Flow}

The present study considers a flow of real fluid streaming past a revolving arrangement with semi-circular geometry having square cross-section. Physical geometry including corresponding notations is illustrated in Fig. 1. The coordinate's $x^{\prime}$ and $y^{\prime}$ axes are considered as the base component and also $z^{\prime}$ is the axial point of the compass. Outer and lower sidewalls of geometry are imposed with different temperatures while the upper and inner sidewalls remaining adiabatically isolated. The temperature of the sidewalls are $T+\Delta T$ while the inmost part is being fixed at $T-\Delta T$, considering $\Delta T$ as positive quantity. The fluid flow is invariant along the central line, which is controlled with different pressures as explained in Fig. 1.

The non-dimensional governing equations the bend pipe is written as:

Continuity equation:

$\frac{\partial u^{\prime}}{\partial r^{\prime}}+\frac{\partial v^{\prime}}{\partial y^{\prime}}+\frac{u^{\prime}}{r^{\prime}}=0$

Momentum equations:

$$
\begin{aligned}
& \frac{\partial u^{\prime}}{\partial t^{\prime}}+u^{\prime} \frac{\partial u^{\prime}}{\partial r^{\prime}}+v^{\prime} \frac{\partial u^{\prime}}{\partial y^{\prime}}-\frac{w^{\prime 2}}{r^{\prime}}=-\frac{1}{\rho} \frac{\partial P^{\prime}}{\partial r^{\prime}}+v\left[\frac{\partial^{2} u^{\prime}}{\partial r^{\prime 2}}+\frac{\partial^{2} u^{\prime}}{\partial y^{\prime 2}}+\frac{1}{r^{\prime}} \frac{\partial u^{\prime}}{\partial r^{\prime}}-\frac{u^{\prime}}{r^{\prime 2}}\right] \\
& \frac{\partial v^{\prime}}{\partial t^{\prime}}+u^{\prime} \frac{\partial v^{\prime}}{\partial r^{\prime}}+v^{\prime} \frac{\partial v^{\prime}}{\partial y^{\prime}}=-\frac{1}{\rho} \frac{\partial P^{\prime}}{\partial y^{\prime}}+v\left[\frac{\partial^{2} v^{\prime}}{\partial r^{\prime 2}}+\frac{1}{r^{\prime}} \frac{\partial v^{\prime}}{\partial r^{\prime}}+\frac{\partial^{2} v^{\prime}}{\partial y^{\prime 2}}\right]+g \beta T^{\prime},
\end{aligned}
$$



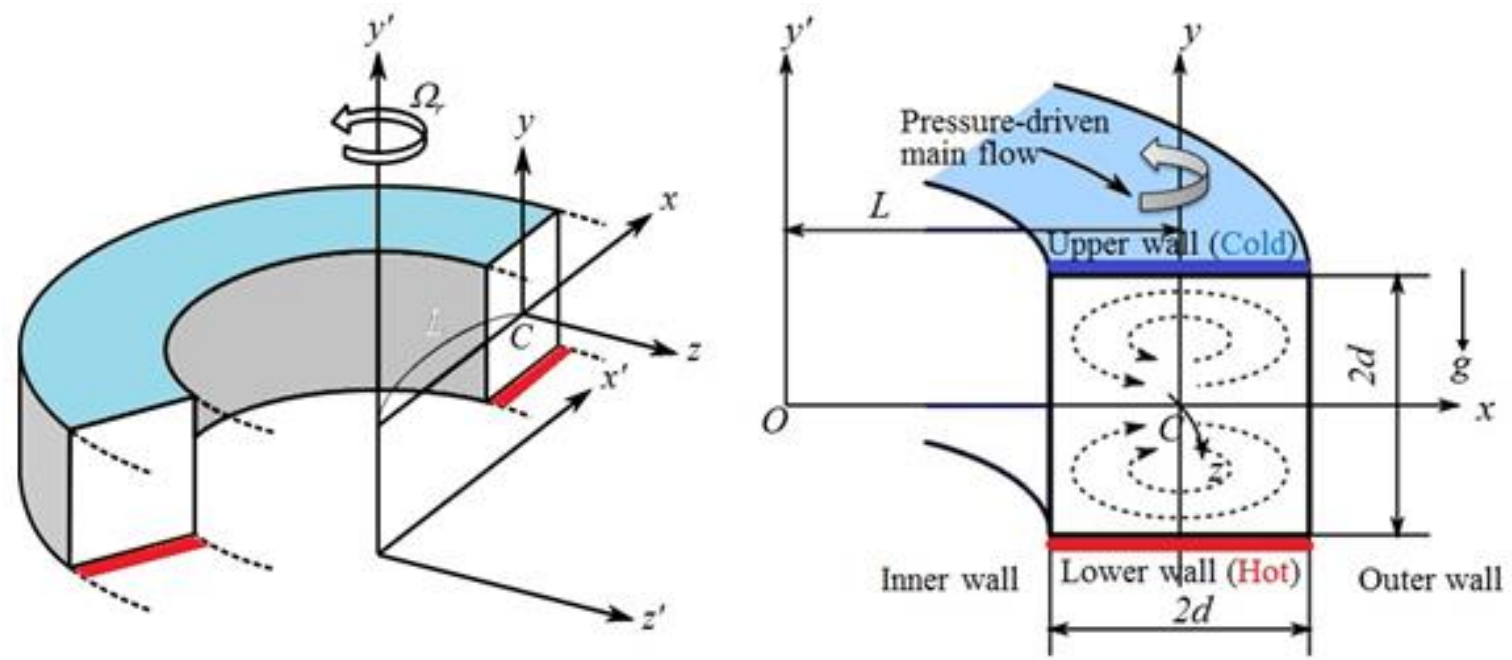

Figure 1. Geometrical view of the bend pipe

$$
\frac{\partial w^{\prime}}{\partial t^{\prime}}+u^{\prime} \frac{\partial w^{\prime}}{\partial r^{\prime}}+v^{\prime} \frac{\partial w^{\prime}}{\partial y^{\prime}}+\frac{u^{\prime} w^{\prime}}{r^{\prime}}=-\frac{1}{\rho} \frac{1}{r^{\prime}} \frac{\partial P^{\prime}}{\partial z^{\prime}}+v\left[\frac{\partial^{2} w^{\prime}}{\partial r^{\prime 2}}+\frac{\partial^{2} w^{\prime}}{\partial y^{\prime 2}}+\frac{1}{r^{\prime}} \frac{\partial w^{\prime}}{\partial r^{\prime}}-\frac{w^{\prime}}{r^{\prime 2}}\right],
$$

Energy equation:

$$
\frac{\partial T^{\prime}}{\partial t^{\prime}}+u^{\prime} \frac{\partial T^{\prime}}{\partial r^{\prime}}+v^{\prime} \frac{\partial T^{\prime}}{\partial y^{\prime}}=\kappa\left[\frac{\partial^{2} T^{\prime}}{\partial r^{\prime 2}}+\frac{1}{r^{\prime}} \frac{\partial T^{\prime}}{\partial r^{\prime}}+\frac{\partial^{2} T^{\prime}}{\partial y^{\prime 2}}\right]
$$

Where $r^{\prime}=L+x^{\prime}$, and $u^{\prime}, v^{\prime}$, and $w^{\prime}$ are the dimensional quantities and these velocities are nil at the boundary layer. The other pieces $P^{\prime}$ is the pressure, $T^{\prime}$ is the temperature and $t^{\prime}$ is the time in dimensional sense. The given arrangements $\rho, \mu, \beta, \kappa$ and $g$ having their usual meaning. Dimensional quantities are then converted into non-dimensional component by introducing the characteristic width $d$ and the free stream velocity $U_{0}=\frac{v}{d}$. The quantities have been defined as:

$$
\begin{gathered}
u=\frac{u^{\prime}}{U_{0}}, v=\frac{v^{\prime}}{U_{0}}, w=\frac{\sqrt{2 \delta}}{\mathrm{U}_{\mathrm{O}}} w^{\prime}, x=\frac{x^{\prime}}{d}, \bar{y}=\frac{y^{\prime}}{d}, z=\frac{z^{\prime}}{d} \\
T=\frac{T^{\prime}}{\Delta T^{\prime}}, t=\frac{U_{0}}{d} t^{\prime}, \delta=\frac{d}{L}, P=\frac{P^{\prime}}{\rho U_{0}{ }^{2}}, G=-\frac{\partial P^{\prime}}{\partial z^{\prime}} \frac{d}{\rho U_{0}^{2}}
\end{gathered}
$$

Here $u, v$ and $w$ are the new velocity along $x, \bar{y}$ and $z$ coordinates, consecutively; $t$ is the dimensionless time, $P$ is the pressure, $\delta$ is the curvature. A unique variable $y$ is then put on the $\bar{y}$ axis as $\bar{y}=l y$. Flow field is taken to be homogeneous along the axial path; the one-sided stream function $\psi$ is set up along the $x$ and $y$ lines as:

$u=\frac{1}{1+\delta x} \frac{\partial \psi}{\partial y} \quad$ and $\quad v=-\frac{1}{1+\delta x} \frac{\partial \psi}{\partial x}$

(6)

After that the rudimental mathematical expression for the axial flow $(w)$, secondary flow $(\psi)$ and temperature distribution $(T)$ are calculated from equations (1)-(5) as follows:

$(1+\delta x) \frac{\partial w}{\partial t}=D n-\frac{\partial(w, \psi)}{\partial(x, y)}+\frac{\delta^{2} w}{1+\delta x}+(1+\delta x) \Delta_{2} w-\frac{\delta}{1+\delta x} \frac{\partial \psi}{\partial y} w+\delta \frac{\partial w}{\partial x}$ 


$$
\begin{aligned}
& \left(\Delta_{2}-\frac{\delta}{1+\delta x} \frac{\partial}{\partial x}\right) \frac{\partial \psi}{\partial t}=-\frac{1}{(1+\delta x)} \frac{\partial\left(\Delta_{2} \psi, \psi\right)}{\partial(x, y)}+\frac{\delta}{(1+\delta x)^{2}} \times\left[\frac{\partial \psi}{\partial y}\left(2 \Delta_{2} \psi-\frac{3 \delta}{1+\delta x} \frac{\partial \psi}{\partial x}+\frac{\partial^{2} \psi}{\partial x^{2}}\right)-\frac{\partial \psi}{\partial x} \frac{\partial^{2} \psi}{\partial x \partial y}\right] \\
& +\frac{\delta}{(1+\delta x)^{2}} \times\left[3 \delta \frac{\partial^{2} \psi}{\partial x^{2}}-\frac{3 \delta^{2}}{1+\delta x} \frac{\partial \psi}{\partial x}\right]-\frac{2 \delta}{1+\delta x} \frac{\partial}{\partial x} \Delta_{2} \psi+w \frac{\partial w}{\partial y}+\Delta_{2}^{2} \psi-G r(1+\delta x) \frac{\partial T}{\partial x} \\
& \frac{\partial T}{\partial t}+\frac{1}{(1+\delta x)} \frac{\partial(T, \psi)}{\partial(x, y)}=\frac{1}{\operatorname{Pr}}\left(\Delta_{2} T+\frac{\delta}{1+\delta x} \frac{\partial T}{\partial x}\right)
\end{aligned}
$$

The dimensionless quantities $D n$, Dean number; Gr, Grashof number and $P r$, Prandtl number, which contain in Eqs. (7) to (9) are expressed as:

$D n=\frac{G d^{3}}{\mu v} \sqrt{\frac{2 d}{L}}, G r=\frac{\beta g \Delta T d^{3}}{v^{2}}, \operatorname{Pr}=\frac{v}{\kappa}$

The boundary conditions for $w$ and $\psi$ are:

$w( \pm 1, y)=w(x, \pm 1)=\psi( \pm 1, y)=\psi(x, \pm 1)=\frac{\partial \psi}{\partial x}( \pm 1, y)=\frac{\partial \psi}{\partial y}(x, \pm 1)=0$

also boundary condition for $T$ is considered invariant near the walls as follows:

$T(x, 1)=1, T(x,-1)=-1, T(x, \pm 1)=y$

After forming the above governing equations and the boundary conditions, they have to satisfy the symmetry condition with respect to the horizontal wall $y=0$ and the solution which satisfies the symmetric condition is defined as symmetric solution. The symmetric conditions are explained as:

$\left.\begin{array}{l}w(x, y, t) \Rightarrow w(-x, y, t), \\ \psi(x, y, t) \Rightarrow-\psi(-x, y, t), \\ T(x, y, t)=-T(-x, y, t)\end{array}\right\}$

\section{Numerical Calculation}

\subsection{Method of numerical calculation}

To solve the governing equations ( 7 to 9 ) with using the boundary conditions (10-11) numerically, spectral method is applied. In this method, the variables are expanded with a series of functions adopting with Chebyshev polynomials. The functions $\varphi_{n}(x)$ and $\psi_{n}(x)$ are defined as:

$$
\begin{aligned}
& \beta_{n}(x)=\left(1-x^{2}\right) \quad P_{n}(x),
\end{aligned}
$$

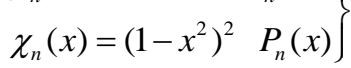

Where $C_{n}(x)=\cos \left(n \cos ^{-1}(x)\right)$ is the first kind Chebyshev polynomial of $n^{\text {th }}$ order. Also, $w(x, y, t), \psi(x, y, t)$ and $T(x, y, t)$ are set by using the function $\beta_{n}(x)$ and $\chi_{n}(x)$ as:

$$
\left.\begin{array}{c}
w(x, y, t)=\sum_{m=0}^{M} \sum_{n=0}^{N} w_{m n}(t) \beta_{m}(x) \beta_{n}(y) \\
\psi(x, y, t)=\sum_{m=0}^{M} \sum_{n=0}^{N} \psi_{m n}(t) \chi_{m}(x) \chi_{n}(y) \\
T(x, y, t)=\sum_{m=0}^{M} \sum_{n=0}^{N} T_{m n}(t) \beta_{m}(x) \beta_{n}(y)+x-y
\end{array}\right\}
$$

Here $m=1, \ldots, M+1$ and $n=1, \ldots, N+1$ where $M$ and $N$ are regarded as grid size. The coefficients of $w_{m n}$, $\psi_{m n}$ and $T_{m n}$ are discarded into the Eq. (7) - (9) and finally the collocation procedure is employed with the coordinates as given. To solve steady solutions, the collocation points $\left(x_{i}, y_{j}\right)$ are assumed as:

$$
\left.x_{i}=\cos \left[\pi\left(1-\frac{i}{M+2}\right)\right], y_{j}=\cos \left[\pi\left(1-\frac{j}{N+2}\right)\right]\right\}
$$


Where $i=1, \ldots, M+1$ and $j=1, \ldots, N+1$. These collocation points are also combined with the NewtonRaphson iteration method or arc-length method. The equation of the arc-length is:

$$
\sum_{m=0}^{M} \sum_{n=0}^{N}\left\{\left(\frac{d w_{m n}}{d s}\right)^{2}+\left(\frac{d \psi_{m n}}{d s}\right)^{2}+\left(\frac{d T_{m n}}{d s}\right)^{2}\right\}=1
$$

This equation is calculated with initial guesses. The initial guesses of the equations are considered as $s+$ $\Delta s$ from point $s$ and can be written as:

$$
\left.\begin{array}{c}
w_{m n}(s+\Delta s)=w_{m n}(s)+\frac{d w_{m n}(s)}{d s} \Delta s \\
\psi_{m n}(s+\Delta s)=\psi_{m n}(s)+\frac{d \psi_{m n}(s)}{d s} \Delta s \\
T_{m n}(s+\Delta s)=T_{m n}(s)+\frac{d T_{m n}(s)}{d s} \Delta s
\end{array}\right\}
$$

The convergence test is confirmed by accepting $\varepsilon_{p}<10^{-10}$, where $p$ refers to repetition of the loop and $\varepsilon_{p}$ is defined as:

$$
\varepsilon_{p}=\sum_{m=0}^{M} \sum_{n=0}^{N}\left[\left(w_{m n}^{(p+1)}-w_{m n}^{p}\right)^{2}+\left(\psi_{m n}^{(p+1)}-\psi_{m n}^{p}\right)^{2}+\left(T_{m n}^{(p+1)}-T_{m n}^{p}\right)^{2}\right]
$$

It is also noted that time dependent derivatives are settled as zero to find out the steady solutions. On the other hand, to investigate the unsteady flow behavior, time derivatives are also taken and used the same collocation points with applying the Cranck-Nicolson together with Adam Bashforth method in equation (7) to (9).

\subsection{Grid efficiency}

To explore grid efficiency give the assurance about the numerical algorithm. In this study, grid accuracy is checked for different grid size. Here, grid sizes are represented by $M$ and $N$ respectively. Table 1 represents the efficiency of the algorithm of governing equations for different grid size. As the present study is based on the bend pipe, so $M$ and $N$ are equal. It is obtained that the values of resistance coefficient and axial flow are not fluctuated enough for increasing or decreasing grid sizes. So, the grid size $M=N=20$ have been taken for present numerical calculation.

Table 1. The values of $\lambda$ and $w(0,0)$ for various $M$ and $N$ at $D n=500, G r=500 \& \delta=0.2$.

\begin{tabular}{cccc}
\hline$M$ & $N$ & $\Lambda$ & $w(0,0)$ \\
\hline 16 & 16 & 0.364843916150 & 179.865392315 \\
18 & 18 & 0.364861316538 & 179.966629534 \\
$\mathbf{2 0}$ & $\mathbf{2 0}$ & $\mathbf{0 . 3 6 4 8 8 4 6 8 6 3 3 3}$ & $\mathbf{1 8 0 . 0 4 7 3 2 9 2 9 3}$ \\
22 & 22 & 0.364873113927 & 180.112646909 \\
24 & 24 & 0.364873081751 & 180.160046771 \\
\hline
\end{tabular}

\subsection{Resistance coefficient}

Resistance coefficient is a dimensionless parameter which is also known as hydraulic resistance coefficient. This parameter is widely used in fluids engineering such as curved duct, elliptical duct, pipe etc. Mathematically, it can be defined as:

$$
\frac{d_{1}^{*}-d_{2}^{*}}{\Delta z^{*}}=\frac{\lambda}{d_{h}^{*}} \frac{1}{2} \rho\left\langle w^{*}\right\rangle^{2}
$$

Where $\langle w\rangle=\frac{\sqrt{2 \delta} d\left\langle w^{*}\right\rangle}{v}$ and the symbols with an asterisk indicate dimensional quantity, the symbol \langle\rangle is the average quantity and $d_{h}{ }^{*}$ is addressed as the hydraulic diameter of the bend pipe. The dimensional mean velocity $\left\langle w^{*}\right\rangle$ is calculated from: 
$\left\langle w^{*}\right\rangle=\frac{v}{4 \sqrt{2 \delta} d} \int_{-1}^{1} d x \int_{-1}^{1} \bar{w}(x, y, t) d y$

By considering $d_{1}{ }^{*}-d_{2}{ }^{*} / \Delta z^{*}=G$, and simplifying the above equations the non-dimensional resistance coefficient can be written as:

$\lambda=\frac{4 \sqrt{2 \delta} D n}{\langle w\rangle^{2}}$,

So it can be easily said from the equation that the resistance coefficient is inversely proportional to the flow coefficient which illustrates how much flow capacity an obstruction allows.

\subsection{Nusselt Number}

The Nusselt number $(\mathrm{Nu})$ is required to calculate the heat transfer between cooled and heated sidewalls. There are two ways to investigate $\mathrm{Nu}$, such as $\mathrm{Nu}$ for both steady solutions and unsteady solutions. The $\mathrm{Nu}$ for the steady solution can be defined as,

$$
\left.\begin{array}{l}
N u_{c}=\frac{1}{2} \int_{-1}^{1}\left[\frac{\partial T}{\partial x}\right]_{x=-1} d y \\
N u_{h}=\frac{1}{2} \int_{-1}^{1}\left[\frac{\partial T}{\partial x}\right]_{x=1} d y
\end{array}\right\}
$$

and for the unsteady solutions can be identified as:

$$
\left.\begin{array}{l}
N u_{\tau_{c}}=\frac{1}{2} \int_{-1}^{1}\left\langle\left\langle\left.\frac{\partial T}{\partial x}\right|_{x=-1}\right\rangle\right\rangle d y \\
N u_{\tau_{h}}=\frac{1}{2} \int_{-1}^{1}\left\langle\left\langle\left.\frac{\partial T}{\partial x}\right|_{x=1}\right\rangle\right\rangle d y
\end{array}\right\}
$$

The notation $\langle\langle\rangle\rangle$ in unsteady solutions used for the average over a time interval. The another notation $\tau$ is defined as for one period, but when the solution is chaotic it takes as an appropriate time interval.

\section{Result and Discussion}

\subsection{Steady Solution with linear stability analysis}

With the path continuation technique, two steady branches are found for Grashof number, $G r=100,500$ \& 1000 and Dean number, $100 \leq D n \leq 1000$ as displayed in Figs. 2 (a), (b) \& (c) respectively. The steady branches are consecutively designated as "Branch 1 (1 $1^{\text {st }}$ branch)" and "Branch 2 ( $2^{\text {nd }}$ branch)." The $1^{\text {st }}$ and $2^{\text {nd }}$ branch is shown in Fig. 2 by red and blue solid line where only the $1^{\text {st }}$ branch is the only one that covers the entire Dean number set. Without turning, the $1^{\text {st }}$ branch initiates at $e$ of $D n=100$ and terminates at $d$ of $D n=$ 1000.The $2^{\text {nd }}$ branch starts from $d$ of $D n=1000$ and the continuation path turns at $b$ of $D n=659.02,686.78 \&$ 710.75 (for Grashof number, $G r=100,500 \& 1000$, consecutively) and terminates at $c$ of $D n=1000$. It is observed that the $1^{\text {st }}$ branch for $G r=100,500 \& 1000$ has overlapped each other. Alternatively, the region of the $2^{\text {nd }}$ branch is curtailed with raising the Grashof number.

Now, stable and unstable points of the steady branches are computed for two-dimensional case. Here, $z$ axis is regarded as the independent axis and the perturbation technique is applied to find out the linear stability. On account of this, the perturbation of flow velocity $(w(x, y), \psi(x, y))$ and isotherms $(T(x, y))$ are considered as eigenvalue problem which is calculated by combining function expansion and collocation technique. The time dependence perturbation is addressed as, $e^{\sigma t}$, where $\sigma=\sigma_{r}+i \sigma_{i}$. Here $\sigma_{r}$ and $\sigma_{i}$ represent the real and imaginary part respectively. If only a single positive value is contained in $\sigma_{r}$ then it shows linear unstable. On the other hand all the values of $\sigma_{r}$ bear negative value then it is linear stable. It is also noted that there is obtained two types of perturbation in the unstable area including oscillatory and monotonically. When $\sigma_{i} \neq 0$, then the unstable area is oscillatory otherwise the unstable area is monotonically. After investigating the linear stability for $G r=100,500 \& 1000$, it is observed that only the first branch shows linear stability among the two branches. For $G r=100,500 \& 1000$; the linear stable points are bound between $100 \leq D n \leq 846.13,209.57 \leq$ 
$D n \leq 1000$ and $414.16 \leq D n \leq 1000$, respectively as shown in Table 2 . The unstable regions are also shown in Figs. 2 (a), (b) \& (c) by thick black solid line. It is demonstrated from the linear stability analysis that linear unstable region at the large $D n$ and small $G r$ has reduced for increasing the $G r$ and a newly unstable region has consisted gradually for small $D n$ and this region has stretched up for rising the $G r$.

Velocity contours (top \& middle) and isotherms (bottom) are visualized in Fig. 3. According to axial flow, the flow velocities push opposite to the inner portion of the bend pipe and generate two high-velocity regions at the broad $\mathrm{Dn}$. The secondary flows narrate that between the necessary range of Dn and Gr, two to four vortex asymmetric flows are produced, with only two vortexes for the $1^{\text {st }}$ branch and two to four vortexes for the $2^{\text {nd }}$ branch. It is remarked that a close interaction exists between the flow velocities (axial and secondary flow). For $G r=1000$ and $D n=800$, a pair of high-velocity regions are occurred at the top and bottom wall which is stronger than the previous flow patterns. For this reason, the secondary flows have consisted of four vortex secondary flow.

(a)

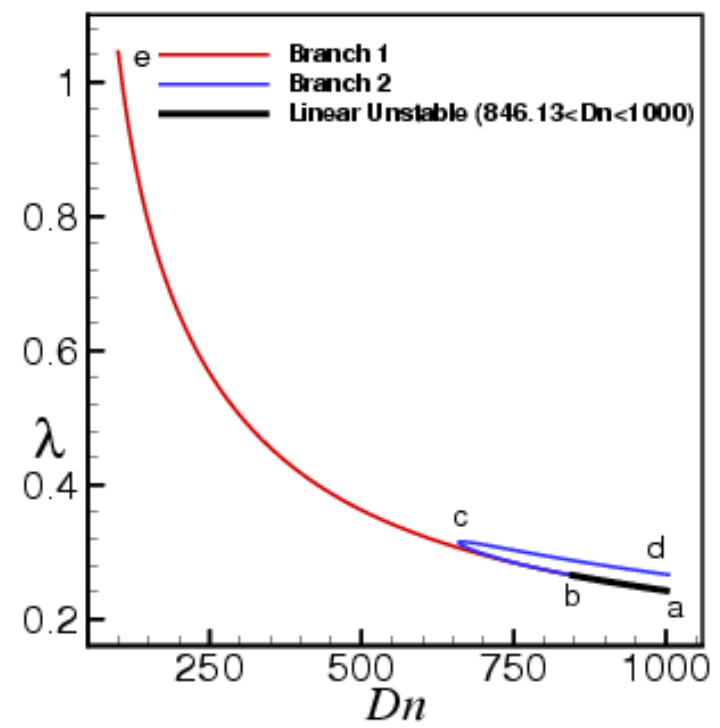

(b)

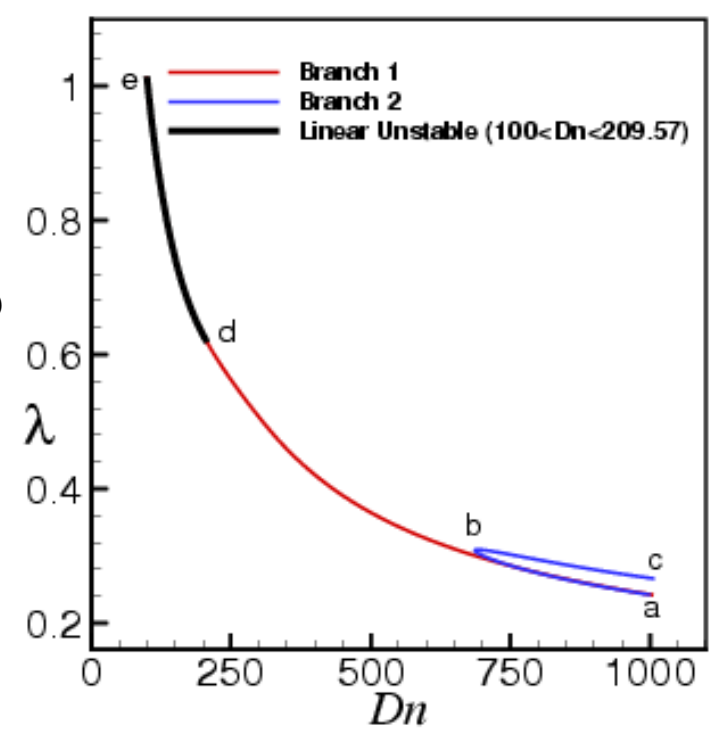

(c)

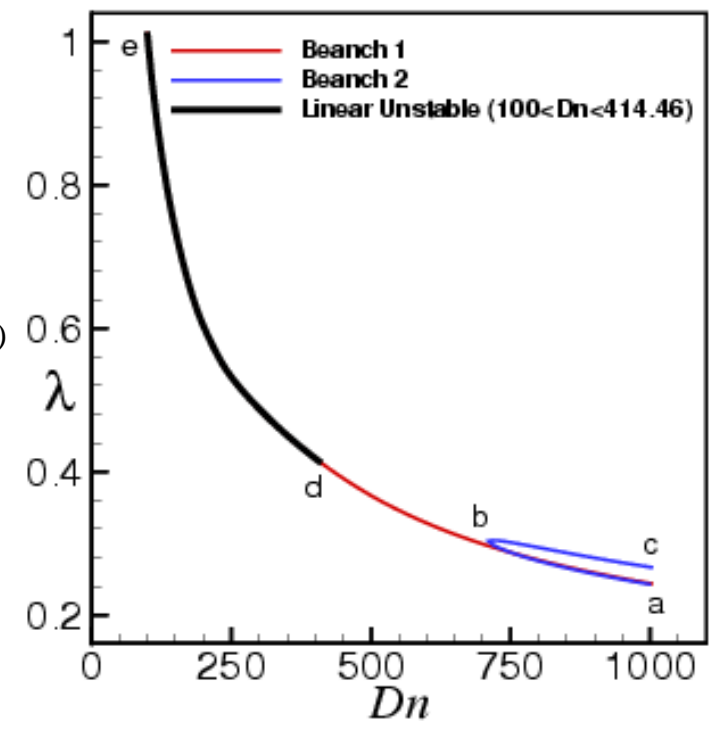

Figure 2. Steady solution branches; for (a) $G r=100$, (b) $G r=500$, (c) $G r=1000$. 
Table 2. Linear Stability analysis for $G r=100,500 \& 1000$.

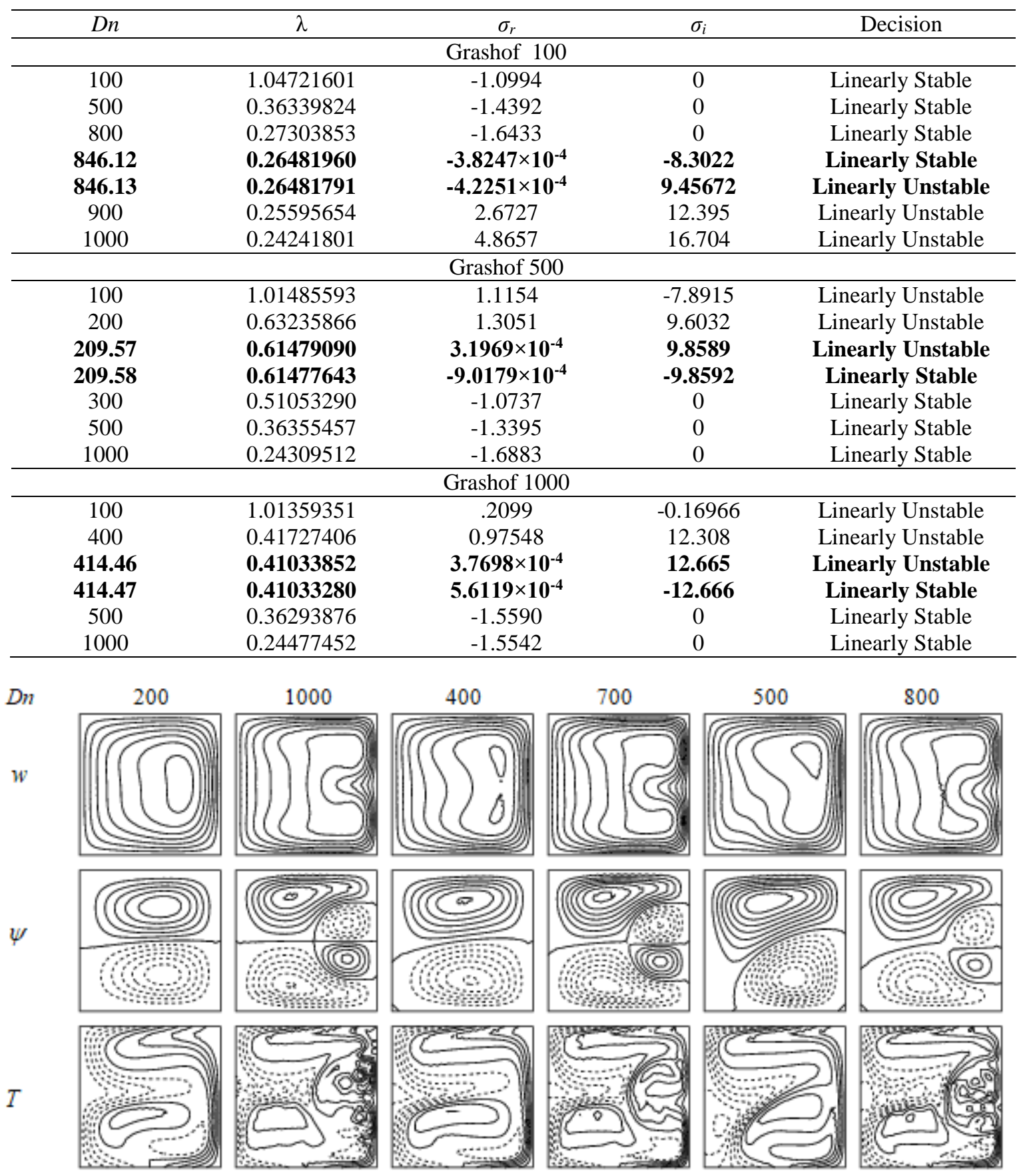

Figure 3. Velocity contours (top \& middle) and isotherms (bottom); for $G r=100,500 \& 1000$.

\subsection{Unsteady solution}

To examine the nonlinear characteristic of stable and unstable solutions, time evaluation findings are accomplished for $G r=100$ and $D n=100,500 \& 1000$ as shown in Figs. 4(a) \& 5(a). The time evolution graphs reveal stable solution for $D n=100$ and 500, and multi-periodic oscillation for $D n=1000$. As shown in Fig. 4(a), the value of $\lambda$ decreases as $D n$ increases and consequently the time evolution graphs transform into multiperiodic flows. We also spread out the power spectrum density (PSD) plot of the simulation data for $D n=1000$ on the Frequency vs. power spectrum density surface in Fig. 5(b) to get a good insight of the flow. PSD reveals not only the dynamic frequency line spectrum and its harmonics but also other line spectra and their harmonics 
with weak amplitudes, indicating that the oscillation in Fig. 5(a) is multi-periodic. Axial velocity, stream function and isotherms for $D n=100,500$ and 1000 are seen in Figs. 4(b) and 6 respectively. The axial velocity reveals for $D n=100$ that the velocity has not sufficiently potent/ strong but it has pushed opposite to the outer part of the pipe from $D n=500$ and finally, it has produced a pair of high-velocity regions at $D n=1000$. The streamlines of secondary flows illustrate that only two-vortex and four vortex flows are generated for steadystate and multi-periodic oscillation respectively. A robust bond among the flow velocities (axial and secondary flow) is also noticed. From Fig. 6, when the axial flows have constructed with a couple two high-velocity areas at the top and bottom part, the secondary flows have shown four vortex solutions. It is said that to represent the flow velocities and isotherms, the grid space $\Delta \psi=1.29, \Delta T=0.33$ and $\Delta w=13.0$ are taken. For all figures in this investigation, the corresponding values of flow velocities and isotherms, solid $(\psi \geq 0)$ and dotted lines $(\psi$ $<\quad 0)$ indicate counter-clockwise and clockwise direction consecutively.

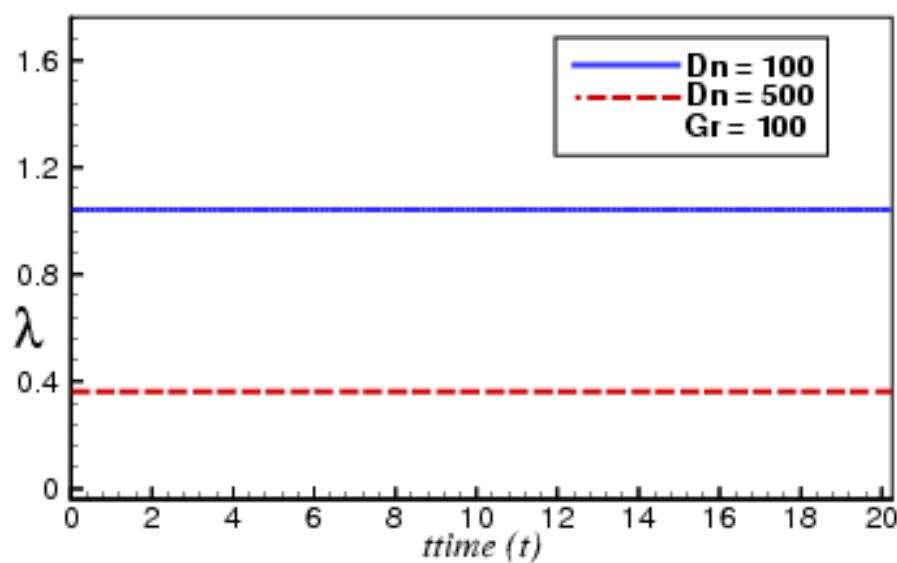

(a)

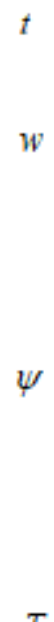

$T$
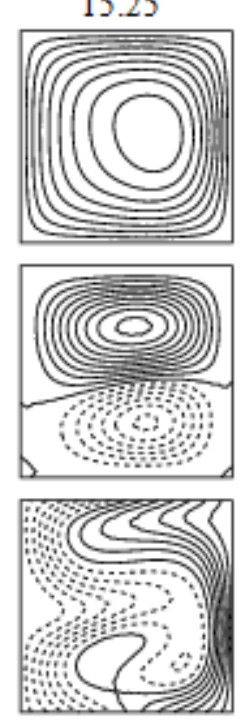

100
19.85
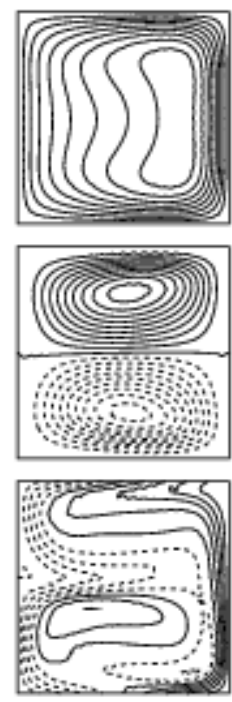

(b)

500

Figure 4. (a) Transient solution, (b) Velocity contours (top \& middle) and isotherms (bottom); for $D n=100 \& 500$ and $G r=100$.

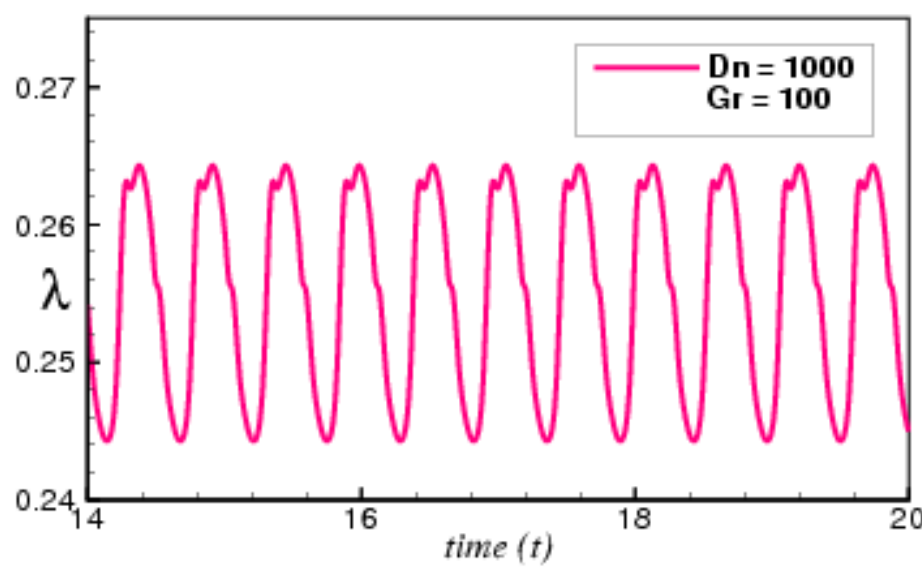

(a)

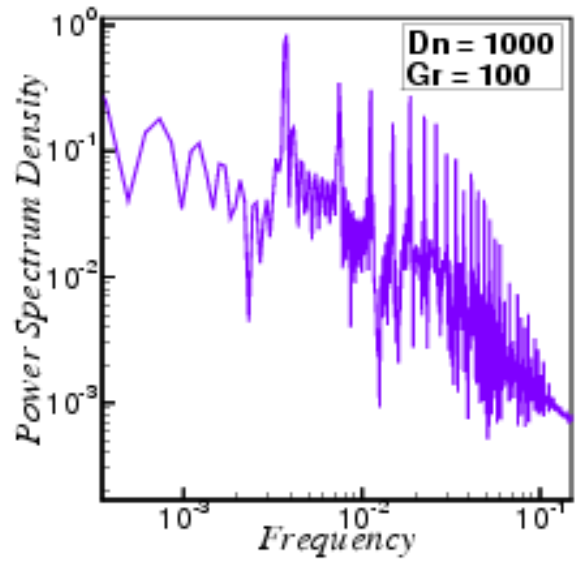

(b)

Figure 5. (a) Transient solution, (b) Power spectrum density; for $D n=1000$ and $G r=100$. 

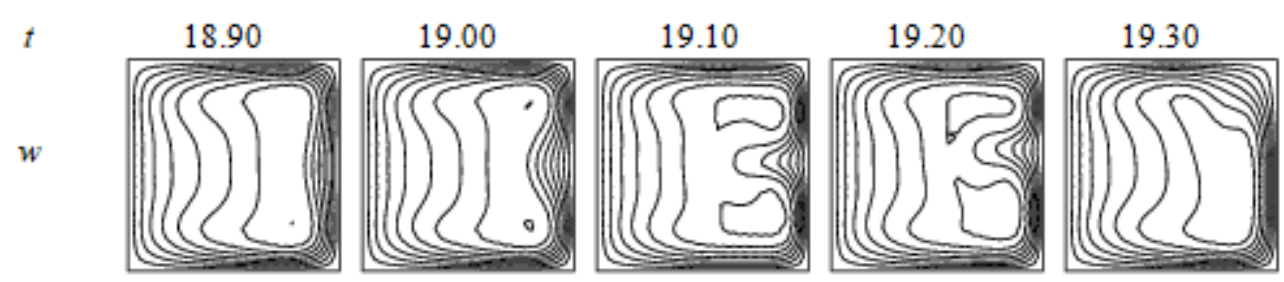

19.40
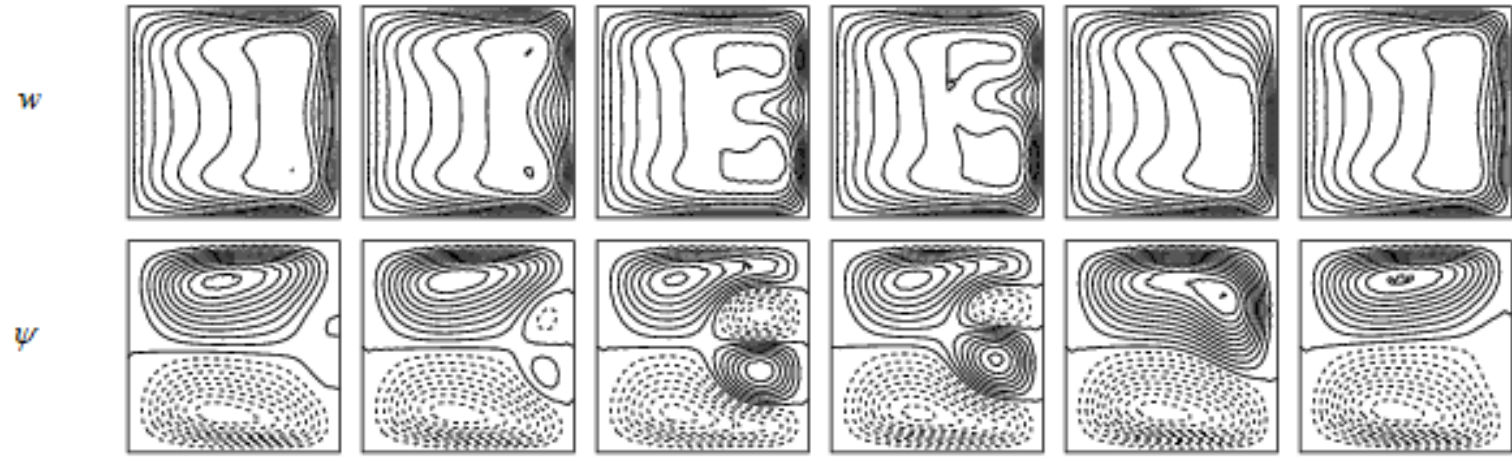

$T$
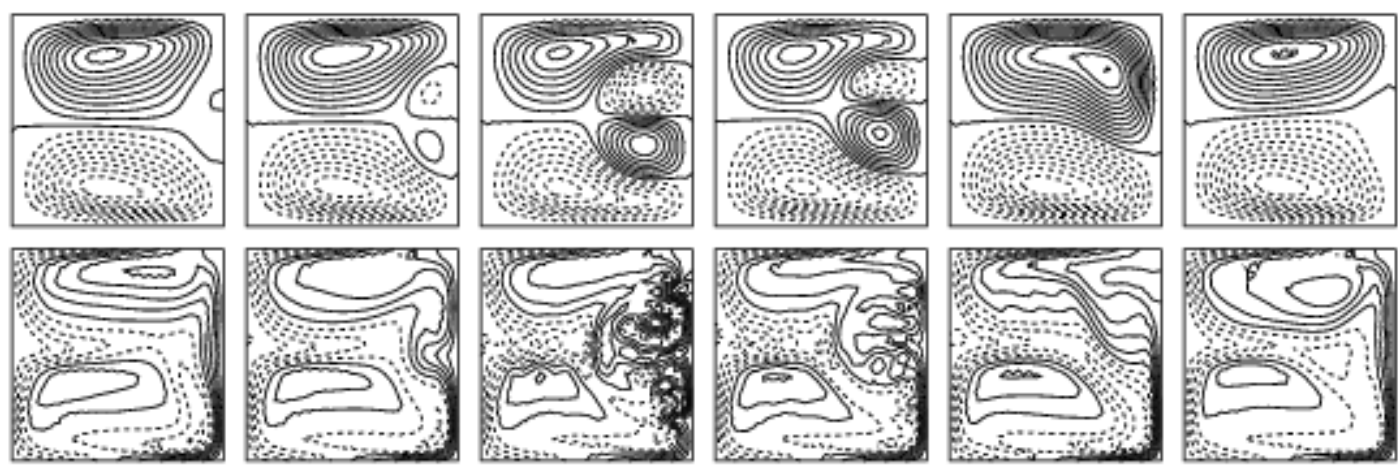

Figure 6. Velocity contours (top \& middle) and isotherms (bottom); for $D n=1000$ and $G r=100$.

After that time history of $\lambda$ has been carried out for $G r=500$ and $D n=100,500 \& 1000$ as represented in Figs. 7(a) \& 9(a). It is detected that the regular oscillation transforms toward the steady-state for increasing the Dean number which is reversed to above exploration. For $D n=100$, the PSD supports the periodic flow, as exposed in Fig. 7 (b). Figs. 8 and 9 (b) visualize the flow velocity and the isotherms for $D n=100,500$ and 1000. The time-dependent solution has formed an asymmetric two-vortex configuration, where isotherms are consistent with the vortex structure.

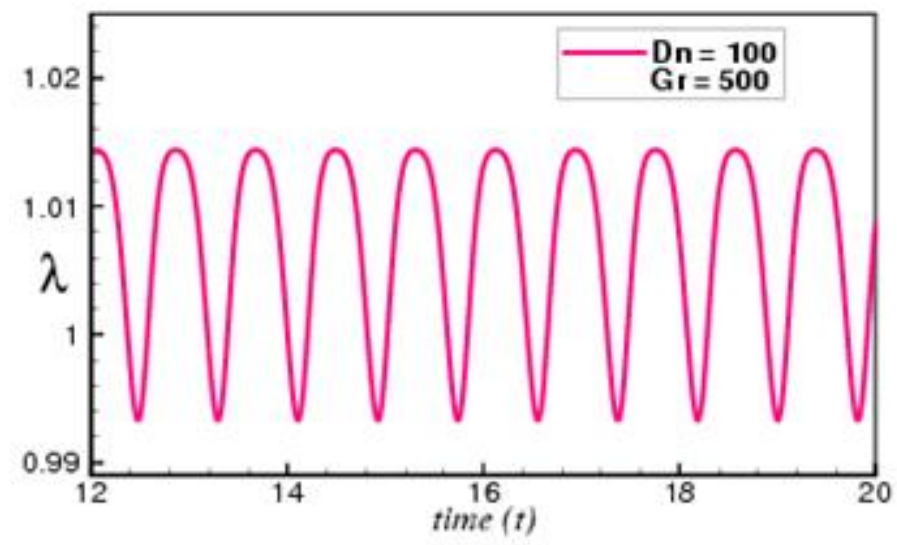

(a)

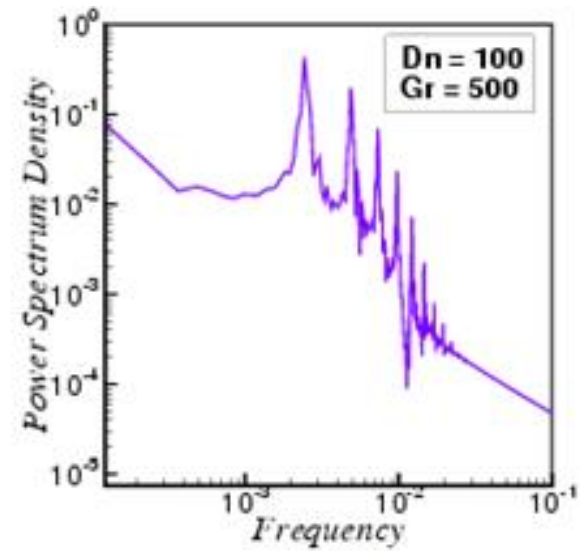

(b)

Figure 7. (a) Transient solution, (b) Power spectrum density; for $D n=100$ and $G r=500$. 
$t$

$w$
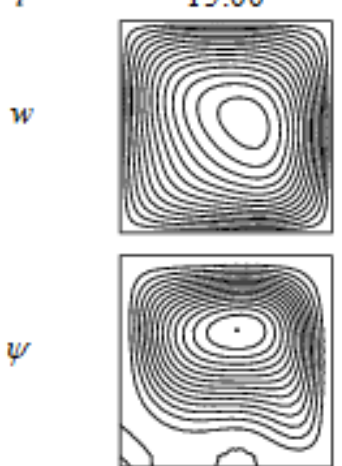

$T$

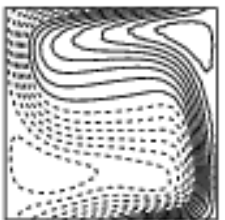

15.10
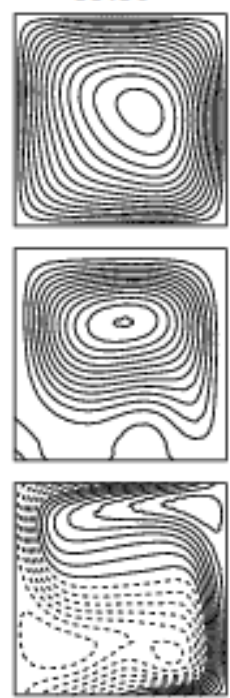

15.20
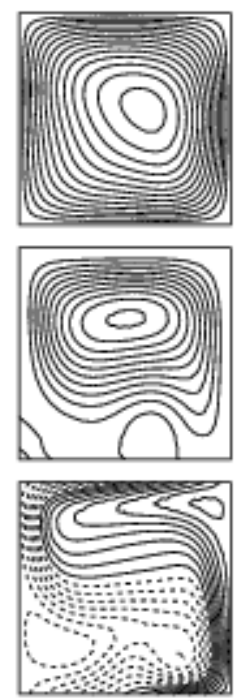

15.30
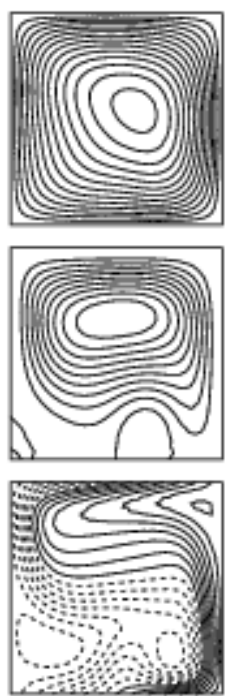

15.40
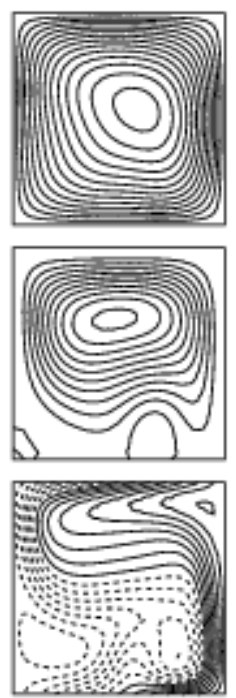

15.50
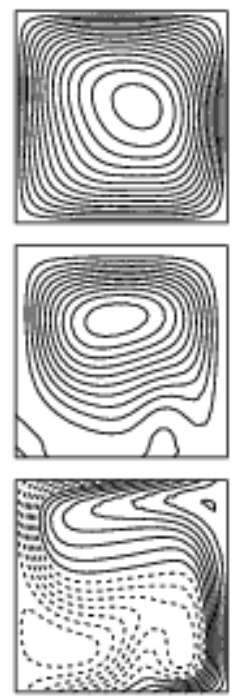

Figure 8. Velocity contours (top \& middle) and isotherms (bottom); for $D n=100$ and $G r=500$.

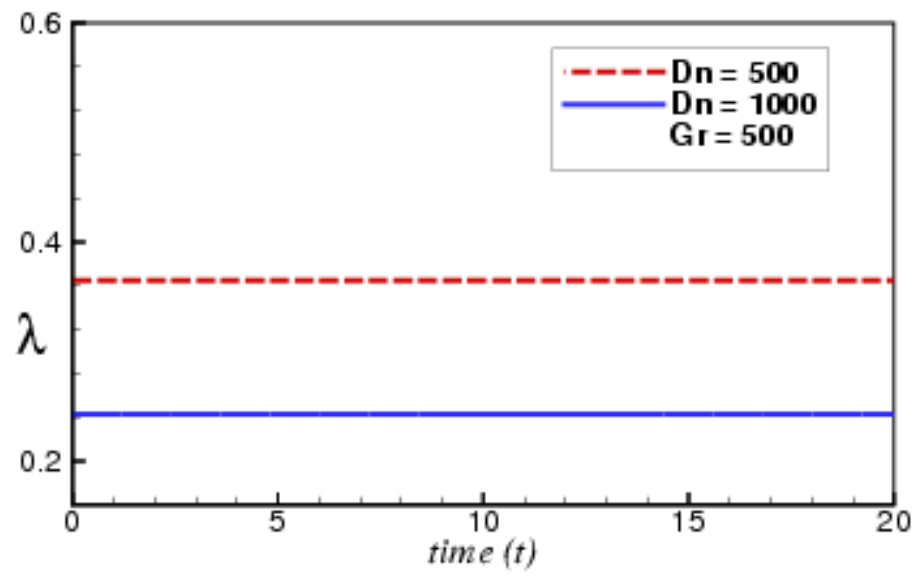

(a)
W
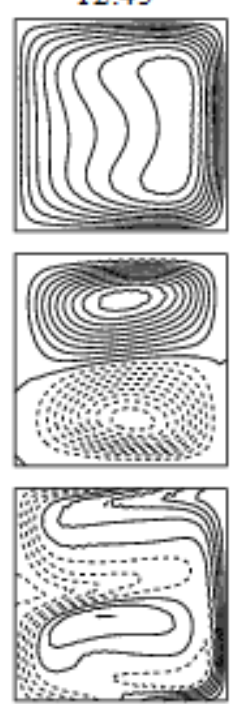

500
19.65
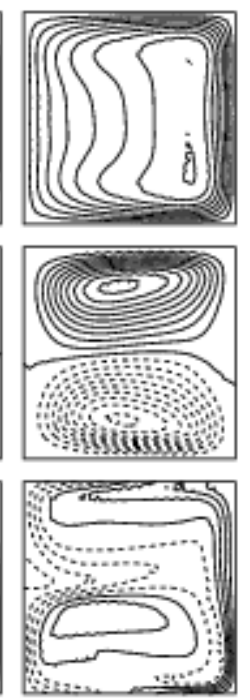

(b)

1000

Figure 9. (a) Transient solution, (b) Velocity contours (top \& middle) and isotherms (bottom); for $D n=100 \& 500$ and $G r=500$.

If $G r$ is even raised higher, i.e. at $G r=1000$, the regular oscillation flows change gradually to the stable solution which is also justified from table 2 . From the stability analysis, it is illustrated that the oscillating flows are continued form $D n=100$ to $D n=414.14$ so the steady-state solutions start from $D n$ $=414.15$. Figs. 10(a) and 12(a) expressed the time-dependent solution for $D n=100$ and $D n=500 \& 1000$ respectively. PSD is moreover disclosed for $D n=100$ in Fig. 10(b), and the velocity contours and the isotherms are interpreted in Figs. 11 and 12(b) for $D n=100$ and $D n=500 \& 1000$ consecutively. It is suggested that the time-dependent solution appears to be nearly identical at $\mathrm{Gr}=500$ and $\mathrm{Gr}=1000$ but there are some differences in the flow characteristics. It can be shown that increasing the $G r$ upsurges the number of dysfunctional regions in relation to the $D n$. But the oscillation of the flow has become weaker for high $G r$ at low $D n$ which has been found by the PSD analysis as seen in Fig. 7. 


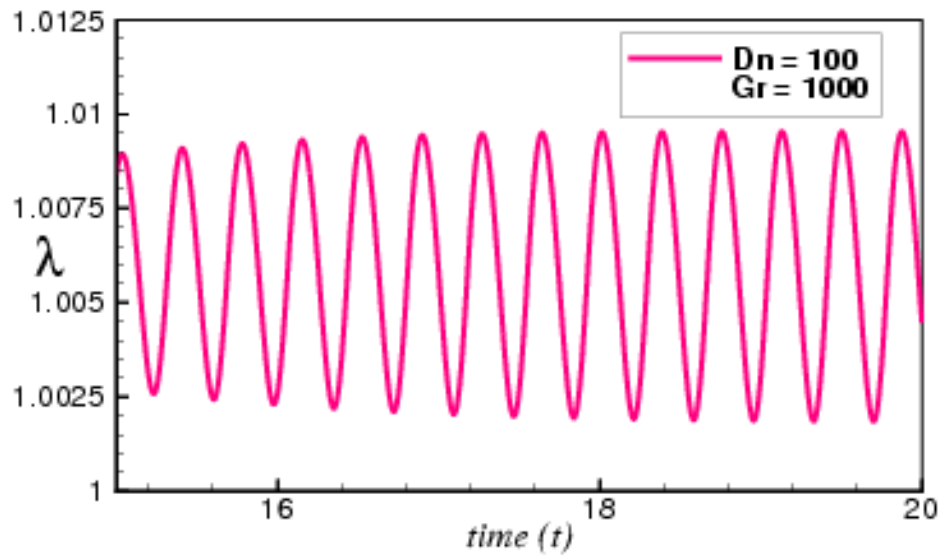

(a)

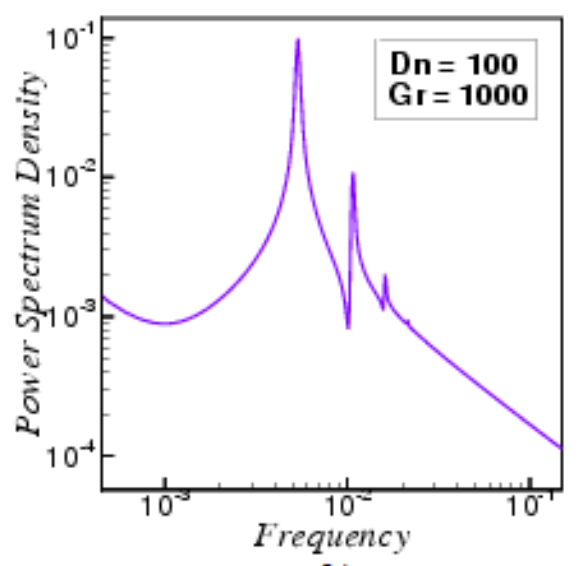

(b)

Figure 10. (a) Transient solution, (b) Power spectrum density; for $D n=100$ and $G r=1000$.
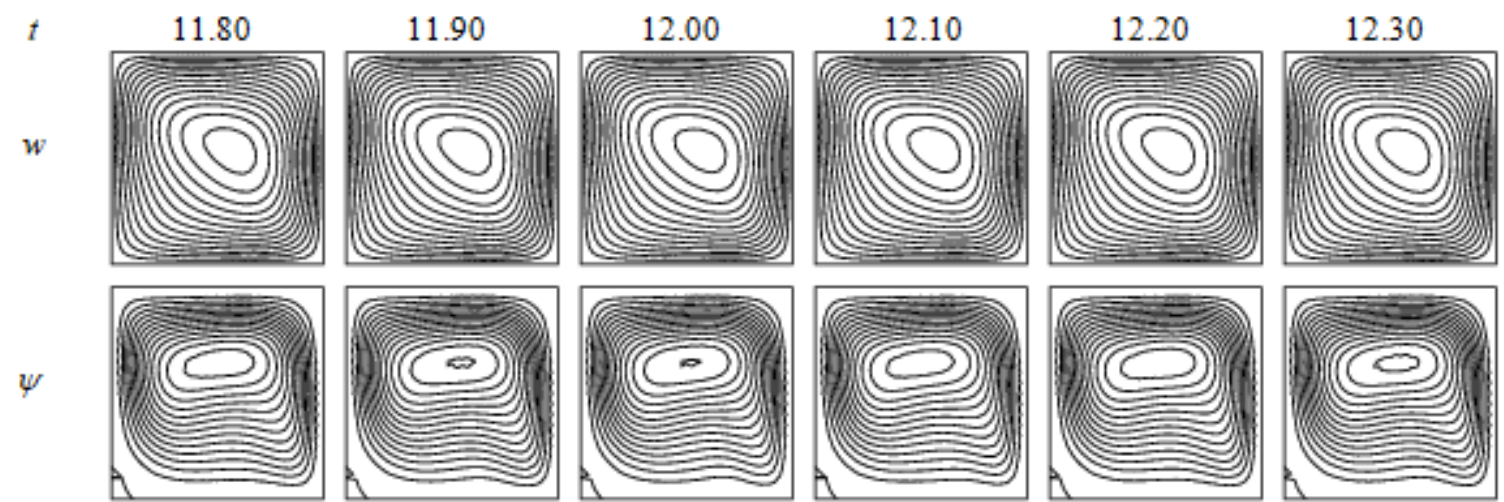

$T$
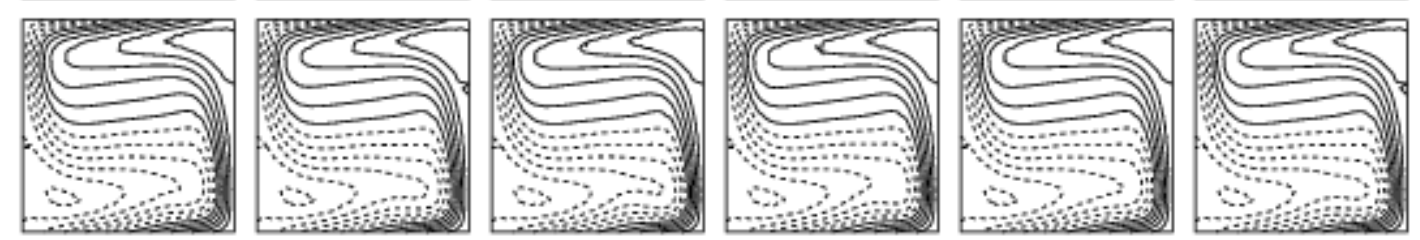

Figure 11. Velocity contours (top \& middle) and isotherms (bottom); for $D n=100$ and $G r=$ 1000. 


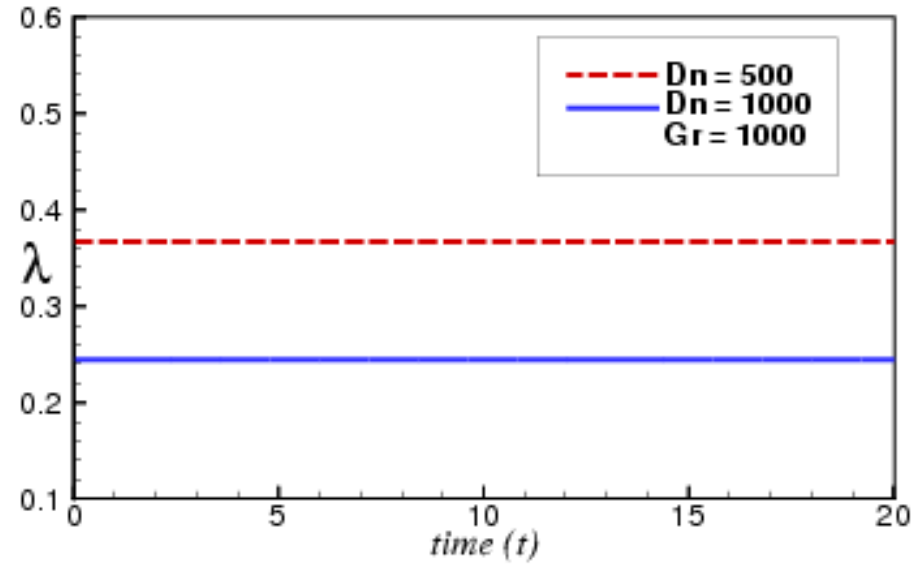

(a) $t$

W
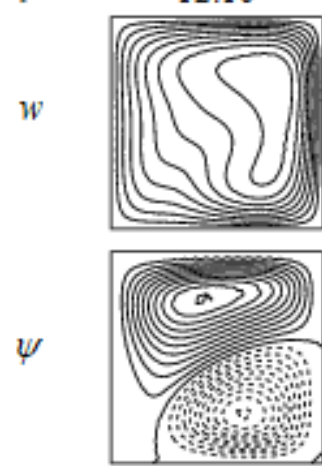

$T$

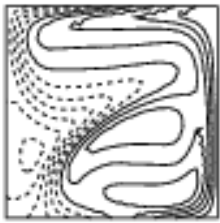

500
17.50
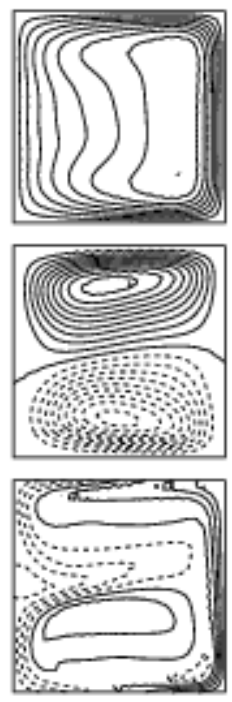

(b) 1000

Figure 12. (a) Transient solution, (b) Velocity contours (top \& middle) and isotherms (bottom); for $D n=100 \& 500$ and $G r=1000$.

\subsection{Energy transfer}

Here, energy transfer through bend pipe is calculated for several Grashof number $(G r=100,500 \& 1000)$ and exposed in Figs. 13 (a), (b) \& (c) respectively. In the figures, the black solid lines are represented for the cooled sidewalls and red solid lines are addressed for the heated sidewalls which are obtained from the steady branches (section 4.1) and calculated by equation (23) where the equation represents that the $N u$ 's are conducted as a directory of horizontal energy distribution from the sidewalls to the fluid. In this study, two steady solution branches are found (section 4.1). Herein, only the first branch is taken to visualize the energy transfer because the first branch shows linear stability and covers the entire Dn among the steady branches. On the other hand, the circle and cross symbols in the figures are obtained from the time average of $N u$ enumerated by equation (24). To be more explicitly, time-dependent solutions for a fixed Dean number are found out at first and then the average of that data is computed and traced in the Figs. 13 (a), (b) \& (c). However, after depicting the heat transfer for three different Grashof number, $G r=100,500 \& 1000$, different types of behavior is found. For $G r=100,500 \& 1000$, the solid lines and symbols in steady states solution have coincided with each other when a steady-state solution occurs. But, when the steady-state solution converts into periodic, heat transfer in $G r=100$ at heated sidewalls has enhanced more than the cooled sidewalls. Alternatively, energy distribution reduced at the heated sidewalls at $G r=1000$ than the cooled sidewalls. This is generated due to the advection and fluid mixing in the pipe. So, it can be said that because of the increasing Dean number, the heat is generated more and creates better flow separation at the outer and bottom sidewalls of the pipe. 

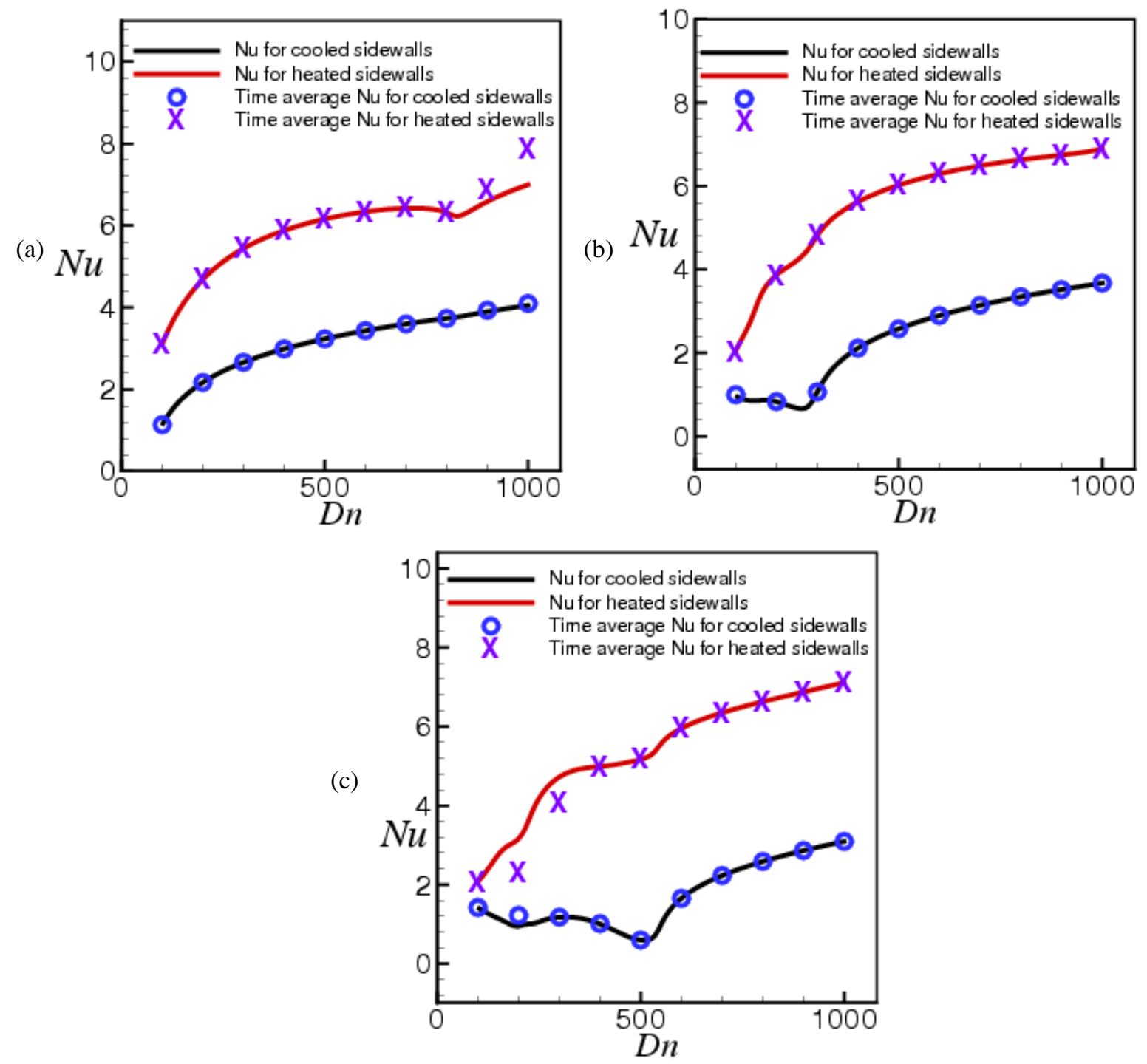

Figure 13. Energy transfer; for (a) $G r=100$, (b) $G r=500$, (c) $G r=1000$.

\subsection{Numerical Validation with experimental results}

In this section, a validation between the numerical and experimental results is performed. Many authors experimentally explored the flow characteristics for different Dean and Taylor numbers or for changing the time. One of the scholars of them, Wang and Yang (2005) disclosed the secondary fluid flow characteristics for non-rotating curved duct. Here, we compared our numerical data to Wang and Yang's (2005) experimental findings. At first, a scheme of the bend duct is constructed and then some smoke are injected through the pipe with constant $D n=300$ which was monitored by a pressure regulator. The smokes were produced by burning Chinese incenses near the settling chamber's inlet. After injecting smoke, some photos of the smoke flow were captured from the $270^{\circ}$ inlet for observing the flow characteristics at various times. For comparison, we have transformed the value of the parameters that are same as Wang and Yang (2005) data. Then we have also run the program with fixing the Dean number $(D n=300)$ and found some secondary flows for different times which are shown in Fig. 14. It is demonstrated from the figure that the secondary flow found by the numerical outcomes is totally same as the experimental results. 

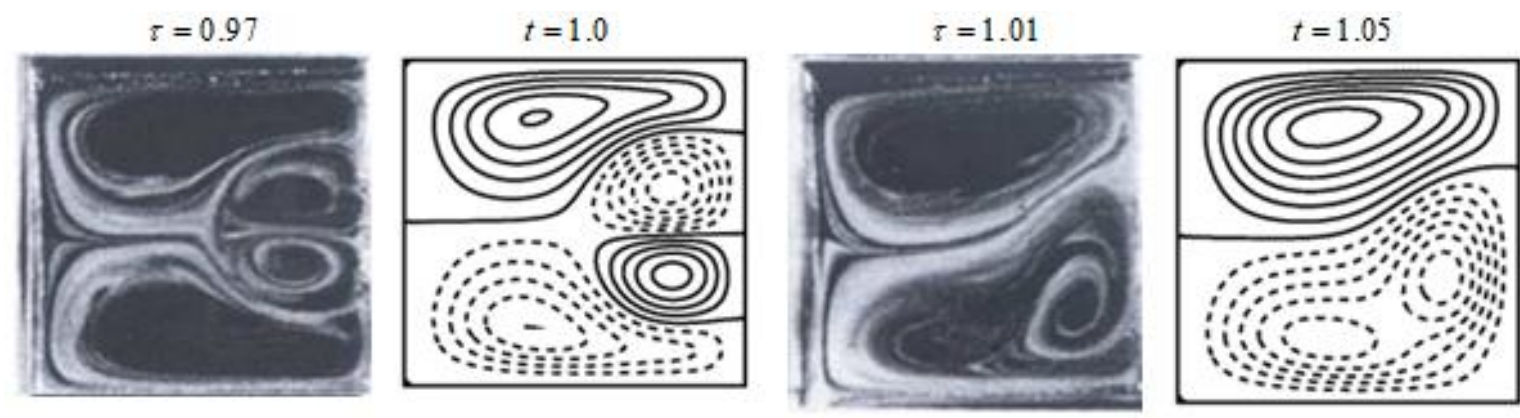

Figure 14. Validation of the numerical results (found by authors, right of the figure) and the experimental outcome (found by Wang and Yang (2005), left of the figure) at $D n=300$.

\section{Conclusion}

In the ongoing exploration, two-dimensional calculations of the flow through the bend square pipe are examined for an extensive range of $D n$ and $G r, 100 \leq D n \leq 1000 ; 100 \leq G r \leq 1000$ with fixed curvature, $\delta=$ 0.2 . At first, two steady branches are found from steady solution where there is no bifurcation connection between them. It is seen that the region of the $2^{\text {nd }}$ branch is reduced for raising the Grashof numbers. Linear stability for different Grashof numbers is also tested and only the first steady branch has shown the linear stability. The time-dependent solutions disclose various types of flow instabilities. For $G r=100$ to $G r=400$, the scheme of the flow behaviors have "steady-state $\rightarrow$ periodic and for increasing the flow behavior are reversed, i.e., it reveals periodic $\rightarrow$ steady-state between $500 \leq G r \leq 1000$. To better assurance about the oscillating behavior, power spectrums are further drawn. Fow velocities (axial and secondary flow) and isotherms are demonstrated while seconday velocity for 2 nd branch represents that two vortex solutions become four vortexes due to the turning of the branch. The flow behavior of the unsteady solutions said that two vortexes have existed for the steady-state solution whereas only two- and four-vortex solutions have been traced out for the periodic/multi-periodic flows. Energy transfer through the pipes reveals that the heat is increased at periodic flows more than steady states flows. Since the flows oscillate, as a result, the fluid particles collide properly as well as increase the overall heat throughout the fluid particles.

\section{References}

Alam, M.M., Ota, M., Ferdows, M., Islamv, M.N., Wahiduzzaman, M., \& Yamamoto, K. (2007). Flow through a rotating helical pipe with a wide range of the Dean number. Arch. Mech., 59(6), 501-517.

Anand, R.B., \& Sandeep, R. (2010). Effect of Angle of Turn on Flow Characteristics of Y-Shaped Diffusing Duct Using CFD. IEEE conference proceedings, Frontiers in Automobile and Mechanical Engineering, 295300. https://doi.org/10.1109/FAME.2010.5714819

Arpino, F., Cortellessa, G., \& Mauro, A. (2015). Transient Thermal Analysis of Natural Convection in Porous and Partially Porous Cavities. Numerical Heat Transfer, Part A, 67, 605-631. https://doi.org/10.1080/10407782.2014.949133

Arvanitisa, K.D., Bourisa, D., \& Papanicolaou, D. (2018). Laminar flow and heat transfer in U-bends: The effect of secondary flows in ducts with partial and full curvature. International Journal of Thermal Sciences, 130, 70-93. https://doi.org/10.1016/j.ijthermalsci.2018.03.027

Bhuyan, D., \& Giri, A. (2021). Heat transfer and second law analysis of turbulent flow mixed convection condensation inside a vertical channel. International Journal of Heat and Mass Transfer, 165, 120658. https://doi.org/10.1016/j.ijheatmasstransfer.2020.120658

Bu, H.X., Tan, H.J., Chen, H., \& He, X.M. (2015). Investigation on Secondary Flow Characteristics in a Curved Annular Duct with Struts. Flow Turbulence Combust, 97(1), 27-41. https://doi.org/10.1007/s10494-0159674-5 
Canton, J., Örlü, R., Chin, C., Hutchins, N., Monty, J., \& Schlatter, P. (2016). On Large-Scale Friction Control in Turbulent Wall Flow in Low Reynolds Number Channels. Flow Turbulence Combust, 97, 811-827. https://doi.org/10.1007/s10494-016-9723-8

Chanda, R.K., Hasan, M.S., Alam, M.M., \& Mondal, R.N. (2020). Hydrothermal behavior of transient fluid flow and heat transfer through a rotating curved rectangular duct with natural and forced convection. Mathematical Modelling of Engineering Problems, 7(4), 501-514. https://doi.org/10.18280/mmep.070401

Chandratilleke, T.T., Nadim, N., \& Narayanaswamy, R. (2013). Analysis of Secondary Flow Instability and Forced Convection in Fluid Flow through Rectangular and Elliptical Curved Ducts. Heat Transfer Engineering, 34(14), 1237-1248. https://doi.org/10.1080/01457632.2013.777249

Chen, L. Cai, J., Lv, K., Yang, X., Chen, S., \& Houa. Y. (2020). Distributed Joule-Thomson effects and convective heat transfer of high-pressure argon gas flow in helically coiled mini-tubes. Applied Thermal Engineering, 181, 115955. https://doi.org/10.1016/j.applthermaleng.2020.115955

Czajkowski, C., Nowak, A.I., \& Pietrowicz, S. (2020). Flower Shape Oscillating Heat Pipe - A novel type of oscillating heat pipe in a rotary system of coordinates - An experimental investigation. Applied Thermal Engineering, 179, 115702. https://doi.org/10.1016/j.applthermaleng.2020.115702

Dean, W.R. (1927). Note on the motion of fluid in a curved pipe. Philos Mag., 4(20), 208-223. https://doi.org/10.1080/14786440708564324

Dolon, S.N., Hasan, M.S., Lorenzini, G., \& Mondal, R.N. (2021). A computational modeling on transient heat and fluid flow through a curved duct of large aspect ratio with centrifugal instability. The European Physical Journal Plus, 136, 382. https://doi.org/10.1140/epjp/s13360-021-01331-0

Dolon, S.N., Hasan, M.S., Ray, S.C., \& Mondal, R.N. (2019). Vortex-structure of secondary flows with effects of strong curvature on unsteady solutions through a curved rectangular duct of large aspect ratio. AIP Conference Proceedings, 2121, 050004. https://doi.org/10.1063/1.5115891

Fiola, C., \& Agarwal, R.K. (2015). Simulation of Secondary and Separated Flow in Diffusing S Ducts. Journal of Propulsion and Power, 31(1), 180-191. https://doi.org/10.2514/1.B35275

Fomicheva, M., Müller, W.H., Vilchevskaya, E.N., \& Bessonov, N. (2019). Funnel flow of a navier-stokesfluid with potential applications to micropolar media. facta universitatis Series: Mechanical Engineering, 17(2): 255 - 267. https://doi.org/10.22190/FUME190401029F

Gao, Y., Feng, Y., Tanga, K., \& Hrnjaka, P. (2020). Experimental study on forced convection hydraulic and thermal characteristics in a dimpled flat duct. Applied Thermal Engineering,181, 115921. https://doi.org/10.1016/j.applthermaleng.2020.115921

Gelfgat, A. (2020), Instability of steady flows in helical pipes. Physical Review Fluids, 5, 103904. https://doi.org/10.1103/PhysRevFluids.5.103904

Ghobadi, M., \& Muzychka, Y.S. (2015). A Review of Heat Transfer and Pressure Drop Correlations for Laminar Flow in Curved Circular Ducts. Heat Transfer Engineering, 37(10), 815-839. https://doi.org/10.1080/01457632.2015.1089735

Hasan, M.S., \& Dolon, S.N., Chakraborty, H.S., Mondal, R.N., Lorenzini, G. (2021). Numerical Investigation on Flow Transition through a Curved Square Duct with Negative Rotation. Journal of Applied and Computational Mechanics (in press), 1-13. https://doi.org/10.22055/JACM.2020.33606.2253

Hasan, M.S., Mondal, R.N., \& Lorenzini, G. (2020). Physics of bifurcation of the flow and heat transfer through a curved duct with natural and forced convection. Chinese Journal of Physics, 67: 428-457. https://doi.org/10.1016/j.cjph.2020.07.004

Hasan, M.S., Islam, M.S., Badsha, M.F., Mondal, R.N., \& Lorenzini G. (2020). Numerical Investigation on the Transition of Fluid Flow Characteristics Through a Rotating Curved Duct. International Journal of Applied Mechanics and Engineering, 25(3), 45-63. https://doi.org/10.2478/ijame-2020-0034

Hasan, M.S., Mondal, R.N., \& Lorenzini, G. (2020). Coriolis force effect in steady and unsteady flow characteristics with convective heat transfer through a curved square duct. International Journal of Mechanical Engineering, 5(1), 1-39. 
Hasan, M.S., Mondal, R.N., \& Lorenzini, G. (2019). Centrifugal Instability with Convective Heat Transfer through a Tightly Coiled Square Duct. Mathematical Modelling of Engineering Problems, 6(3), 397-408. https://doi.org/10.18280/mmep.060311

Hasan, M.S., Islam, M.M., Ray, S.C., \& Mondal, R.N. (2019). Bifurcation structure and unsteady solutions through a curved square duct with bottom wall heating and cooling from the ceiling. AIP Conference Proceedings, 2121, 050003. https://doi.org/10.1063/1.5115890

Hasan, M.S., Mondal, R.N., \& Lorenzini, G. (2019). Numerical Prediction of Non-isothermal Flow with Convective Heat Transfer through a Rotating Curved Square Channel with Bottom Wall Heating and Cooling from the Ceiling. International Journal of Heat and Technology, 37(3), 710-726. https://doi.org/10.18280/ijht.370307

Hasan, M.S., Mondal, R.N., Kouchi, T., \& Yanase, S. (2019). Hydrodynamic instability with convective heat transfer through a curved channel with strong rotational speed. AIP Conference Proceedings, 2121, 030006. https://doi.org/10.1063/1.5115851

Hashemi, A., Fischer, P.F., \& Loth, F. (2018). Direct numerical simulation of transitional flow in a finite length curved pipe. Journal of Turbulence, 19(8), 664-682. https://doi.org/10.1080/14685248.2018.1497293

Islam, M.N., Ray, S.C., Hasan, M.S., \& Mondal, R.N. (2019). Pressure-driven flow instability with convective heat transfer through a rotating curved rectangular duct with differentially heated top and bottom walls. AIP Conference Proceedings, 2121, 030011. https://doi.org/10.1063/1.5115856

Kim, Y.I., Kim, S.H., Hwang, Y.D., \& Park, J.H. (2011). Numerical investigation on the similarity of developing laminar flows in helical pipes. Nuclear Engineering and Design, 241, 5211-5224. https://doi.org/10.1016/j.nucengdes.2011.09.020

Li, Y., Wang, X., Yuan, S., \& Tan, S.K. (2016). Flow development in curved rectangular ducts with continuously varying curvature. Experimental Thermal and Fluid Science, 75, 1-15. https://doi.org/10.1016/j.expthermflusci.2016.01.012

Li, Y., Wang, X., Zhou, B., Yuan, S., \& Tan, S.K. (2017). Dean instability and secondary flow structure in curved rectangular ducts. International Journal of Heat and Fluid Flow, 68, 189-202. https://doi.org/10.1016/j.ijheatfluidflow.2017.10.011

Lin, T.S., Rogers, S., Tseluiko, D., \& Thiele, U. (2016). Bifurcation analysis of the behavior of partially wetting liquids on a rotating cylinder. Physics of Fluids, 28, 082102. https://doi.org/10.1063/1.4959890

Lin, Z., Zhu, Y., \& Wang, Z. (2017). Local bifurcation of electrohydrodynamic waves on a conducting fluid. Physics of Fluids, 29, 032107. https://doi.org/10.1063/1.4979064

Leong, F.Y., Smith, K.A., \& Wang, C.H. (2009). Secondary flow behavior in a double bifurcation. Physics of Fluids, 21, 043601. https://doi.org/10.1063/1.3100211

Liu, F., \& Wang, L. (2009). Analysis on multiplicity and stability of convective heat transfer in tightly curved rectangular ducts. International Journal of Heat and Mass Transfer, 52, 5849-5866. https://doi.org/10.1016/j.ijheatmasstransfer.2009.07.019

Mechane, W. (2010). Bifurcation and stability analysis of laminar flow in curved ducts. International Journal for Numerical Methods in Fluids, 64(4), 355-375. https://doi.org/10.1002/fld.2147

Mokeddem, M., Laidoudi, H., Makinde, O.D., \& Bouzit, M. (2019). 3D Simulation of Incompressible Poiseuille Flow through $180^{\circ}$ Curved Duct of Square Cross-section under Effect of Thermal Buoyancy. Periodica Polytechnica, Mechanical Engineering, 63(4), 257-269. https://doi.org/10.3311/PPme.12773

Mondal, R.N., Watanabe, T., Hossain, M.A., \& Yanase, S. (2017). Vortex-Structure and Unsteady Solutions with Convective Heat Transfer Trough a Curved Duct. Journal of Thermophysics And Heat Transfer, 31(1), 243-254. https://doi.org/10.2514/1.T4913

Mondal, R.N., Islam, M.Z., Islam, M.M., \& Yanase, S. (2015). Numerical Study of Unsteady Heat and Fluid Flow through a Curved Rectangular Duct of Small Aspect Ratio. Science and Technology Asia, 20(4), 1-20. 
Mondal, R.N., Islam, M.S., Uddin, K., \& Hossain, M.A. (2013). Effects of aspect ratio on unsteady solutions through curved duct flow. Applied Mathematics and Mechanics, 34(9), 1107-1122. https://doi.org/10.1007/s10483-013-1731-8

Mondal, R.N., Kaga, Y., Hyakutake, T., \& Yanase, S. (2007). Bifurcation diagram for two-dimensional steady flow and unsteady solutions in a curved square duct. Fluid Dynamics Research, 39(5), 413-446. https://doi.org/10.1016/j.fluiddyn.2006.10.001

Mondal, R.N., Kaga, Y., Hyakutake, T., \& Yanase, S. (2006). Effects of curvature and convective heat transfer in curved square duct flows. Trans. ASME, Journal of Fluids Engineering, 128(9), 1013-1022. https://doi.org/10.1115/1.2236131

Manesh, B.E., Shahmardan, M.M., Rahmani, H., \& Norouzi, M. (2019). Heterogeneous anisotropic conductive heat transfer in composite conical shells: An exact analysis. International Journal of Heat and Mass Transfer, 144, 118614. https://doi.org/10.1016/j.ijheatmasstransfer.2019.118614

Nazeer, G., Islam, S., Shigri, S.H., \& Saeed, S. (2019). Numerical investigation of different flow regimes for multiple staggered rows. AIP Advances, 9, 035247. https://doi.org/10.1063/1.5091668

Nobari, M.R.H., Ahrabi, B.R., \& Akbari, G. (2009). A numerical analysis of developing flow and heat transfer in a curved annular pipe. International Journal of Thermal Sciences, 48, 1542-1551. https://doi.org/10.1016/j.ijthermalsci.2008.12.004

Norouzi, M., Kayhani, M.H., Nobari, M.R.H., \& Talebi, F. (2011). Analytical Investigation of Viscoelastic Creeping Flow and Heat Transfer Inside a Curved Rectangular Duct. Theoretical Foundations of Chemical Engineering, 45(1), 53-67. https://doi.org/10.1134/S0040579511010052

Rahmani, H., Norouzi, M., Birjandi, A.K., \& Birjandi, A.K. (2019). An exact solution for transient anisotropic heat conduction in composite cylindrical shells. Journal of Heat Transfer, 141(10), 101301. https://doi.org/10.1115/1.4044157

Razavi, S.E., Soltanipour, H., \& Choupani, P. (2015). Second Law Analysis of Laminar Forced Convection in a Rotating Curved Duct. Thermal Science, 19(1), 95-107. https://doi.org/10.2298/TSCI120606034R

Sultana, M.N., Hasan, M.S., \& Mondal, R.N. (2019). A numerical study of unsteady heat and fluid flow through a rotating curved channel with variable curvature. AIP Conference Proceedings, 2121, 030009. https://doi.org/10.1063/1.5115854

Soltanipour, H., Gharegöz, A. \& \& Oskooee, M.B. (2020). Numerical study of magnetic field effect on the ferrofluid forced convection and entropy generation in a curved pipe. Journal of the Brazilian Society of Mechanical Science and Engineering, 42, 135. https://doi.org/10.1007/s40430-020-2218-5

Wang, L., \& Yang, T. (2005). Periodic oscillation in curved duct flows. Physica D, 200, 296-302. https://doi.org/10.1016/j.physd.2004.11.003

Wang, X.K., Li, Y.L., Yuan, S.Q., \& Tan, S.K. (2018). Flow past a near-wall retrograde rotating cylinder at varying rotation and gap ratios. Ocean Engineering, 156, 240-251. https://doi.org/10.1016/j.oceaneng.2018.03.015

Watanabe, T., \& Yanase, S. (2013). Bifurcation Study of Three-Dimensional Solutions of the Curved SquareDuct Flow. Journal of the Physical Society of Japan, 82, 074402, 1-9. https://doi.org/10.7566/JPSJ.82.074402

Yanase, S., Mondal, R.N., \& Kaga, Y. (2005). Numerical Study of Non-Isothermal Flow with Convective Heat Transfer in a Curved Rectangular Duct. International Journal of Thermal Science, 44(11), 1047-1060. https://doi.org/10.1016/j.ijthermalsci.2005.03.013

Yanase, S., Kaga, Y., \& Daikai, R. (2002). Laminar flows through a curved rectangular duct over a wide range of the aspect ratio. Fluid Dynamics Research, 31, 151-183. https://doi.org/10.1016/S0169-5983(02)00103-X

Yang, T., \& Wang, L. (2001). Solution Structure and Stability of Viscous Flow in Curved Square Ducts. Journal of Fluids Engineering, 123(4), 863-868. https://doi.org/10.1115/1.1412457

Zhang, J., Chen, H., Zhou, B., \& Wang, X. (2019). Flow around an array of four equispaced square cylinders. Applied Ocean Research, 89, 237-250. https://doi.org/10.1016/j.apor.2019.05.019. 